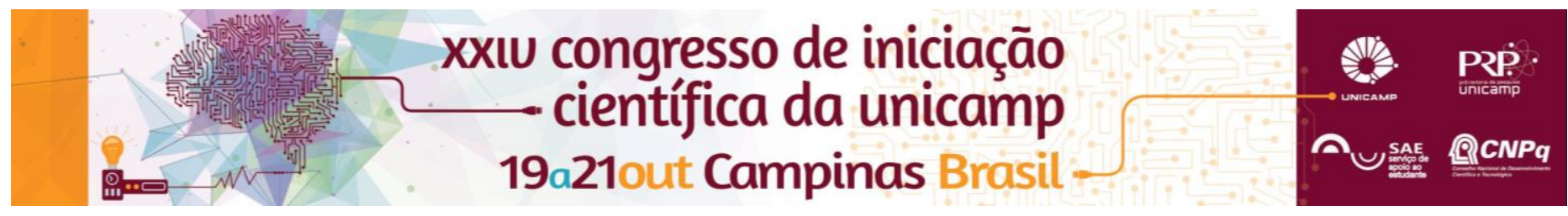

\title{
Finite element simulation of high myopia for the identification and study of retinal pathologies
}

\author{
Tatiana T. S. Mello*, Marco L. Bittencourt, Jorge L. Suzuki, Alexandre C. Souto.
}

\begin{abstract}
We perform a computational simulation of myopia using a human eye model, and analyze the effects on several structures to identify related pathologies. Myopia is a relevant and common clinical condition caused by an increase in the axial length of the eye. Such elongation is correlated with several pathologies like retinal schisis, detachment and posterior staphyloma. Some theories suggest that high myopia is associated with a higher deformations and thinning of the sclera, particularly at the posterior pole of the eye. Highly myopic eyes have decreased amounts of scleral tissue and a reduction of collagen fibril diameter resulting in a thinner sclera. These biomechanical characteristics, combined with the axial elongation are associated with posterior staphyloma formation. Of particular interest, retinal schisis is the result of retinal stretching caused by scleral growth. We use a known eye geometry from the literature to construct an axisymmetric finite element model using ABAQUS. The elongation of the eye is simulated using prescribed displacements according to axial length data from 3 individuals. The simulation results were consistent with the literature, showing scleral thinning and retinal thickening at the posterior pole of the eye. These effects play a role in the formation of staphyloma and retinal schisis, respectively. Moreover, we compared the simulated retinal thickness with the clinical data and observed errors in the range of $2.7 \%-5.2 \%$. The results showed good approximations and we believe that the model can be useful to study myopia and its associated pathologies caused by mechanical loads. Also it would help identifying patients with potential retinal pathologies, aiding ophthalmologists to predict the onset of these pathologies and consequently have more precise estimations for preventive surgical interventions.
\end{abstract}

\section{Key words:}

Finite element analysis, Pathological Myopia, Retinal schisis

\section{Introduction}

A published and validated eye geometry ${ }^{1}$ was used to construct a finite element model with material properties obtained from the literature and a specific set of boundary conditions was used to simulate the eye growth. There is no development regarding the simulation of pathological myopia itself and its influence in the course of retinal pathologies comparing it with real cases for model calibration. So this work focuses on analyzing the effects of the growth on the structures at the posterior pole of the eye and compares it with real patients' cases to understand the retinal pathologies associated with myopia.

\section{Results and Discussion}

We performed two analyses with the elongation of the eye:

- Quantitative analysis: an elongation of $2 \mathrm{~mm}$ was imposed and we analyzed the variation of retinal and scleral thickness in the posterior part of the eye comparing it with the distribution of the strains (Image 1). As studies suggested ${ }^{2}$, we observed that the retina is more mechanically requested and thicker at the posterior pole and thinner in the periphery of myopic eyes comparing to emmetropic ones.

- Qualitative analysis: specific elongations according to patient-specific eyeball lengths were imposed and calculated the error between the simulated and observed retinal thicknesses (Chart 1). The approximation errors showed a good estimate for a problem involving large deformations and nonlinear constitutive relations with material parameters identified from complex materials.

Chart 1. Magnitude of the strain on the macula, observed macula thickness from the patients' exams and simulated macula thickness.

\begin{tabular}{ccccc} 
Patient & $\begin{array}{c}\text { Logarithmic } \\
\text { strain } \\
\text { magnitude }\end{array}$ & $\begin{array}{c}\text { Observed } \\
\text { Macula } \\
\text { thickness } \\
(\mathbf{m m})\end{array}$ & $\begin{array}{c}\text { Simulated } \\
\text { Macula } \\
\text { thickness } \\
(\mathbf{m m})\end{array}$ & $\begin{array}{c}\text { Error } \\
(\%)\end{array}$ \\
\hline 1 & 0.254 & 0.281 & 0.295 & 4.98 \\
2 & 0.310 & 0.295 & 0.303 & 2.71 \\
3 & 0.479 & 0.307 & $0, .23$ & 5.21
\end{tabular}

DOI: 10.19146/pibic-2016-50681

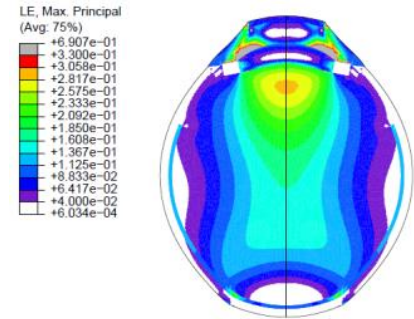

(a) Entire eye

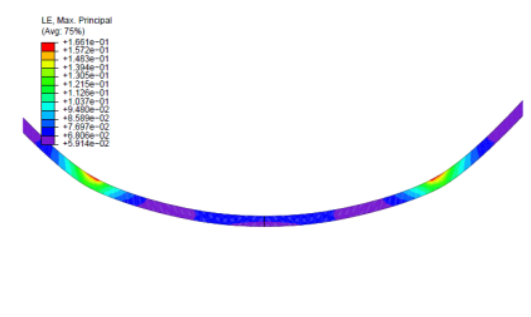

(b) Retina at the posterior pole
Image 1. Maximum principal logarithmic strains (LEMax) in the eye with $2 \mathrm{~mm}$ of displacement. We observe in (b) that the highest strains at the posterior pole are located in the retina, with strains up to 0.16 .

\section{Conclusions}

- The adapted model reproduced the myopic eye consistently, since the mechanical behavior in the posterior region of the eye due to axial elongation was compatible with several studies and with the real patients' cases.

- The retina is the significantly strained and highly susceptible to complications caused by mechanical processes.

- The model can be improved by adding structures of the eye especially to understand the mechanical factors driving degenerative changes, such as staphylomas and retinal schisis.

\section{Acknowledgement}

I'd like to thank Prof.Dr. Marco Lucio Bittencourt, Jorge Luís Suzuki and Dr. Alexandre Souto for their assistance and guidance during the project. And my gratitude towards for PIBIC by the financial support.

${ }^{1}$ LIU, X.; WANG, L. "Mechanism of traumatic retinal detachment in blunt impact: A finite elment study". Journal of Biomechanics, Vol 46, pp 1321-1327.2013.

${ }^{2}$ BENDRE, A. A., "Finite element analysis and preliminary experiments to study the effects of high myopia in macular degeneration". Mechanical Engineering Master's Theses. Northeastern University. Boston. 2009. 